\title{
Producción primaria requerida para sustentar el desembarque de peces pelágicos en Chile
}

\author{
Luis Cubillos S., Sergio Núñez E. y Dagoberto Arcos R. \\ Instituto de Investigación Pesquera \\ Casilla 350, Talcahuano, Chile \\ E-mail: inpesca@arauco.reuna.cl
}

\begin{abstract}
RESUMEN. Se analiza la sustentabilidad de los niveles de desembarque actuales de peces pelágicos en Chile (sobre 7 millones de ton en 1994 y 1995), utilizando como enfoque la capacidad productiva del sistema de surgencia costera de Chile en relación con el desembarque anual promedio de peces pelágicos durante el período 1989-96. Se estima la producción primaria requerida (PPR) por los principales recursos de peces pelágicos explotados sobre la base de las capturas promedio, el nivel trófico de las especies y la eficiencia de transferencia ecotrófica. Además, se analizan indicadores globales del excedente de producción de estas pesquerías a través de la relación existente entre las capturas totales y el esfuerzo de pesca de la flota nacional. Se consideran tres zonas geográficas: norte $\left(18^{\circ} 20^{\prime} \mathrm{S}-29^{\circ} 15^{\prime} \mathrm{S}\right)$, centro-norte $\left(29^{\circ} 15^{\prime} \mathrm{S}-32^{\circ} 10^{\prime} \mathrm{S}\right)$, y centro-sur $\left(32^{\circ} 10^{\prime} \mathrm{S}-39^{\circ} 23^{\prime} \mathrm{S}\right)$. Se asume que el área productiva de la zona costera se extiende hasta 60 millas náuticas fuera de la costa en cada región. La PPR alcanza valores de 137,34 y 614 gCm${ }^{2} \mathrm{anno}^{-1}$ para las zonas norte, centro-norte y centro-sur, respectivamente. El alto valor para la zona centro-sur se debe a los desembarques de Trachurus symmetricus murphyi que, por su naturaleza oceánica y comportamiento migratorio, puede acceder a la producción de otros ecosistemas. Al eliminar este recurso del análisis, la PPR para los restantes recursos pelágicos alcanza a $55 \mathrm{gCm}^{-2} \mathrm{año}^{-1}$ a nivel nacional (17\% de la producción primaria) y a $207 \mathrm{gCm}^{-2} \mathrm{año}^{-1} \mathrm{si} \mathrm{se}$ considera que T. s. murphyi accede parcialmente a la productividad del ecosistema de surgencia. Un análisis de la relación entre las capturas totales y el esfuerzo de pesca indica que la pesquería nacional de peces pelágicos parece haber llegado a un nivel muy cercano al máximo sustentable. En su conjunto, estos análisis sugieren que la capacidad productiva del ecosistema de surgencia costera frente a Chile limita el desembarque nacional de peces pelágicos costeros. El nivel de desembarque promedio observado en el período 1989-96 es sustentable solamente si los excedentes productivos de $T$. s. murphyi son compatibles con la intensidad de su explotación actual por parte de la flota industrial de cerco de la zona centro-sur.
\end{abstract}

Palabras claves: pesquería chilena, desembarque, peces pelágicos, producción, sistema de surgencia.

\section{Primary production required to sustain the chilean pelagic fisheries}

\begin{abstract}
The sustainability of the current landing of pelagic fish in Chile (over 7 millions tons in 1994 and 1995) is analyzed using as a focus the productive capacity of the Chilean coastal upwelling ecosystem in relation to the mean annual catch of pelagic fish during the period 1989-96. The primary production required (PPR) by the main pelagic species is estimated on the basis of the average catch, the trophic level of the resources and the ecotrophic efficiency of transfer. Furthermore, global indicators of the surplus production of these pelagic fisheries are analyzed using the relationship between the total catches and the fishing effort of the national fleet. Three geographic zones are considered: northern $\left(18^{\circ} 20^{\prime} \mathrm{S}-29^{\circ} 15^{\prime} \mathrm{S}\right)$, central-northern $\left(29^{\circ} 15^{\prime} \mathrm{S}-32^{\circ} 10^{\prime} \mathrm{S}\right)$ and central-southern $\left(32^{\circ} 10^{\prime} \mathrm{S}-39^{\circ} 23^{\prime} \mathrm{S}\right)$. The assumption is made that the productive area of the upwelling system extends to 60 nautical miles from the coast in each region. The PPR reaches values of 137,34 and $614 \mathrm{gCm}^{-2}$ year $^{-1}$, respectively for the northern, central-northern and central-southern zones. The high PPR value in the central-southern zone is due to the landings of Trachurus symmetricus murphyi, which given its oceanic nature and highly migratory behaviour can feed in different kinds of ecosystems. Eliminating this species from the analysis, the total PPR for the remaining pelagic resources reaches 55 $\mathrm{gCm}^{-2}$ year $^{-1}$ (17\% of the primary production) and $207 \mathrm{gCm}^{-2}$ year $^{-1}$ by considering that $T$. $s$. murphyi has a partial access
\end{abstract}


to the productivity of the coastal upwelling. The relationship between total catches and fishing effort indicates that the Chilean pelagic fishery has arrived near to the maximum sustainable level. Overall, these analysis suggest that the productive capacity of the Chilean coastal upwelling ecosystem limits the national catch of coastal pelagic fish. The average landing level observed during the period 1989-96 is sustainable only if the surplus production of T. s. murphyi is compatible with the intensity of their current exploitation by the industrial purse seine fleet of the central-southern zone.

Key words: Chilean fisheries, pelagic fish landings, productive capacity, coastal upwelling system.

\section{INTRODUCCION}

La captura total de recursos hidrobiológicos que se desembarca en Chile, ubica al país dentro de las primeras catorce naciones pesqueras del mundo y ocupa el $5^{\circ}$ lugar entre los países que desarrollan su actividad en el Océano Pacífico (FAO, 1990). Los desembarques nacionales se sustentan principalmente en unas pocas especies de peces pelágicos y demersales, típicas de los sistemas de surgencia de borde oriental de los océanos, siendo recursos que se caracterizan por su alta y variable productividad (Bakun y Parrish, 1980; Parrish et al., 1983; LluchBelda et al., 1989; Hutchings, 1992; Yáñez et al., 1997). Los principales recursos de peces pelágicos que se explotan en Chile, y que dan cuenta de aproximadamente el $92 \%$ del desembarque nacional, son: jurel Trachurus symmetricus murphyi, anchoveta Engraulis ringens, sardina Sardinops sagax, caballa Scomber japonicus, y sardina común Strangomera bentincki.

La mayor tasa de crecimiento de los desembarques nacionales de peces ocurrió entre 1978 y 1986, con 420 mil ton por año. A partir de 1987, se observa una disminución de la tasa de crecimiento en concomitancia con algunas fluctuaciones interanuales importantes, aunque la tendencia creciente se mantiene. En 1994, el desembarque nacional alcanzó un máximo histórico de 7,839 millones de ton (excepto las algas), lo que significa un incremento de $29,9 \%$ en relación con 1993. En 1995 el desembarque nacional alcanzó a 7,591 millones de ton, pero en 1996 disminuyó a 6,9 millones de ton, lo que representa una disminución de 11,8\% en relación con 1994.

Los recursos que han contribuido a incrementar el desembarque nacional de peces en 1994 y 1995, han sido principalmente $E$. ringens y, en mayor medida, T. s. murphyi. De acuerdo a lo expuesto, es válido preguntarse acerca de la sustentabilidad de los niveles de captura actuales que el país está obteniendo, con especial énfasis en los principales recursos de peces pelágicos explotados. Debido a que los peces son parte de un complejo sistema de pro- ducción biológica y dado que en Chile una gran fracción de la biomasa de peces que se desembarca proviene de uno de los sistemas de surgencia más productivos del mundo, el objetivo de este trabajo es analizar la capacidad productiva del sistema marino costero de Chile en relación con el desembarque anual de peces pelágicos en el período 1989-96. El enfoque utilizado para analizar la capacidad de carga del sistema consiste básicamente en determinar la magnitud de la producción primaria requerida para sustentar los niveles de desembarque actuales observados (Christensen y Pauly, 1993; Pauly y Christensen, 1995), lo que se complementa con un análisis de las pesquerías de la zona norte y centrosur sobre la base de indicadores biológico pesqueros globales de la pesquería nacional de peces pelágicos.

\section{MATERIALES Y METODOS}

La información de desembarques anuales de las principales especies pelágicas se ha obtenido de los Anuarios Estadísticos de Pesca, editados entre 1958 y 1977 por el Servicio Agrícola y Ganadero, División de Pesca y Caza; y entre 1978 y 1996 por el Servicio Nacional de Pesca. Esta información se analiza particularmente para el período 1989-96, considerando tres grandes zonas: norte $\left(18^{\circ} 20^{\prime} \mathrm{S}\right.$ $\left.29^{\circ} 15^{\prime} \mathrm{S}\right)$, centro-norte $\left(29^{\circ} 15^{\prime} \mathrm{S}-32^{\circ} 10^{\prime} \mathrm{S}\right)$, y centro-sur $\left(32^{\circ} 10^{\prime} \mathrm{S}-39^{\circ} 23^{\prime} \mathrm{S}\right)$. Estas zonas geográficas se basan principalmente en criterios biológicopesqueros, los cuales están dados por las unidades de pesquería definidas y en los patrones de distribución que tienen los recursos pesqueros.

Además, en términos generales, las zonas consideradas presentan características oceanográficas distintas. El área norte se caracteriza por exhibir una muy baja turbulencia, el transporte advectivo fuera de la costa es débil, el ancho de la plataforma continental es moderada, la temperatura superficial del mar exhibe una anomalía cálida casi permanente con una banda de agua fría muy estrecha y costera (Parrish et al., 1983; Bernal et al., 1983); además, a 
lo largo del año ocurre un fuerte predominio anticiclónico, lo que permite que la surgencia inducida por el viento se manifieste prácticamente durante todo el ciclo anual (Pizarro et al., 1994; Barbieri et al., 1995). El área centro-norte (Coquimbo), en cambio, se caracteriza porque la turbulencia es moderada en el verano y alta en el invierno; el transporte fuera de la costa es intenso, la plataforma continental es estrecha ( $\gg 10 \mathrm{Km})$, y en la costa se registran temperaturas frías con filamentos que se extienden varios cientos de $\mathrm{Km}$ fuera de la costa (Parrish et al., 1983). En la zona centro-sur, la surgencia costera es estacional (Ahumada y Chuecas, 1979; Arcos y Navarro, 1986; Arcos, 1987), la turbulencia es alta en la época estival, pero también en la época invernal debido al paso de frentes de mal tiempo por la región, el transporte fuera de la costa es alto en verano, mientras que la extensión de la plataforma continental es moderada a ancha (Ahumada, 1989).

Otra información utilizada para complementar el análisis de capturas, dice relación con la actividad operacional de la flota nacional, particularmente de la flota industrial de cerco, en cuanto a número de viajes totales, capacidad de bodega promedio y acumulada en el período 1965-96. Esta información se obtuvo de Serra (1986), Serra y Tsukayama (1988), Caballero et al. (1992), y datos propios obtenidos de las bases de datos del Instituto de Investigación Pesquera (Hernández y Sepúlveda, 1998), especialmente de la flota industrial de cerco de la zona centro-sur. Con esta información se intenta analizar los cambios en el excedente de producción global a través de la relación existente entre la captura total de peces pelágicos y el esfuerzo de pesca nominal. Dicho análisis se realiza en términos cualitativos más bien que cuantitativos, particularmente debido a lo complejo que podría resultar la estandarización del esfuerzo de la flota nacional para un período de tiempo que cubre aproximadamente 30 años. Por esta razón se utiliza como índice de esfuerzo de pesca el producto entre la capacidad de bodega de la flota y los viajes totales anuales realizados (acarreo nominal).

La producción primaria requerida se calculó sobre la base de la siguiente ecuación (Ryther, 1969; Cushing, 1971; Pauly y Christensen, 1995):

$$
P P R=C \cdot E T^{(N T-1)}
$$

donde PPR es la producción primaria requerida $\left(\mathrm{gCm}^{-2} \mathrm{año}^{-1}\right), \mathrm{C}$ es la captura promedio de peces
$\left(\mathrm{gCm}^{-2} \mathrm{año}{ }^{-1}\right)$ en el período 1989-96, ET es la eficiencia de transferencia ecotrófica, y NT es el nivel trófico del recurso. Para convertir unidades de peso húmedo (ton) a gramos de carbono, se utilizó una razón de 9:1 (Lieth, 1978 fide Pauly y Christensen, 1995). Esta razón es conservativa y se aplica sólo para transformar la captura de peces a gramos de carbono. Se consideró un valor promedio de $10 \%$ para la eficiencia de transferencia ecotrófica en base a los resultados de Christensen y Pauly $(1993,1995)$. Estos autores analizan 48 modelos tróficos de ecosistemas acuáticos, a partir de los cuales obtienen un valor promedio 10,1 (en \%) para la eficiencia de transferencia ecotrófica (desviación estándar de 5,81 y un error estándar de la media de 0,49 ), no observándose tendencias en los valores de ET con los niveles tróficos.

Para el nivel trófico de las principales especies de peces pelágicos que componen el desembarque nacional, entre los $18^{\circ} 20^{\prime} \mathrm{S}$ (Arica) y los $39^{\circ} 37^{\prime} \mathrm{S}$ (Queule), se adoptó los siguientes valores: 2,6 para los clupeiformes (E. ringens, S. bentincki y $S$. sagax); 3,2 para $T$. s. murphyi; y 3,3 para $S$. japonicus, que corresponden a los utilizados por Pauly y Christensen (1995) para estos recursos de peces en áreas de surgencia. A su vez, son consistentes con estudios de alimentación realizados en Chile (Medina, 1990; Miranda et al., 1998; H. Arancibia, com. pers.) y para algunas de las mismas especies en el ecosistema de surgencia de la región centro-norte de Perú (Jarre et al., 1989).

Con el objeto de establecer el nivel porcentual de la PPR respecto de la producción primaria (PP) en cada región, se realizaron estimaciones de la producción primaria (PP) anual sobre la base de la relación general encontrada por Ware (1992) entre la PP $\left(\mathrm{gCm}^{-2} \mathrm{año}{ }^{-1}\right)$ y el Transporte de Ekman (ton $\mathrm{m}^{-}$ $\left.{ }^{1} \mathrm{~s}^{-1}\right)$ de los sistemas de surgencia de California, Benguela y Perú, i.e.

$$
P P=809 \cdot T-81
$$

donde $\mathrm{T}$ es el de Transporte de Ekman.

Los valores de Transporte de Ekman para las tres regiones de estudio se obtuvieron de Parrish et al. (1983), considerando que Arica, Coquimbo y Talcahuano, son localidades representativas de las zonas norte, centro-norte y centro-sur de Chile. Otro dato importante dice relación con el área productiva en estas tres regiones. En la zona centro-sur, el área de influencia de las aguas de la surgencia llega 
a los $32 \mathrm{Km}$ (Ahumada, 1989), pero cuando la surgencia es muy intensa, durante la época estival, la extensión de las aguas surgentes puede tener influencia hasta $80 \mathrm{mn}$ desde la costa (Cáceres y Ar$\cos , 1991)$. En el área comprendida entre los $18^{\circ} \mathrm{S}$ y $24^{\circ} \mathrm{S}$, se identifican persistentemente hasta 8 focos de surgencia, donde las lenguas de agua surgente alcanzan una extensión de 30-40 mn en verano y cerca de $25 \mathrm{mn}$ en invierno; con filamentos formándose en los extremos de las lenguas de agua surgentes que tienen longitudes de 40-60 mn, alcanzando entre 100 y $120 \mathrm{mn}$ de la costa (Barbieri et al., 1995). Ware (1992) utiliza $125 \mathrm{Km}$ desde la costa para limitar el área de influencia de la productividad del sistema de surgencia costera en Perú. En este estudio nosotros utilizamos $60 \mathrm{mn}$ desde la costa $(113 \mathrm{Km})$, para todas las regiones, como el área de influencia más importante asociada a la productividad que la surgencia costera genera.

\section{RESULTADOS}

En 1987 por primera vez ocurre una disminución importante en el desembarque nacional, y desde entonces, hasta 1996, se han observado fluctuaciones interanuales importantes, pero con una tenden- cia que sigue siendo creciente (Figura 1). En 1994 y 1995, se registró los niveles más altos, sobre 7,5 millones de ton), pero en 1996 nuevamente el desembarque disminuye a niveles cercanos a los $7 \mathrm{mi}-$ llones de ton (Figura 1).

El desembarque total nacional es sustentado básicamente por T. s. murphyi, E. ringens, S. bentincki, $S$. japonicus, y $S$. sagax, con un aporte que en promedio alcanza a 91\% en el período 1989-96 (Figura 1). Actualmente, E. ringens es el recurso dominante en las capturas de la zona norte y $T$. s. murphyi el recurso principal en la zona centro-sur.

$\mathrm{El}$ aumento del desembarque nacional de peces pelágicos se debe al desarrollo y evolución del tamaño de la flota nacional, que desde 1989 ha tendido a la estabilización en el número de unidades de pesca (Figura 2a). Los viajes totales, en cambio, muestran una tendencia a disminuir desde cerca de 45 mil viajes en 1989 hasta cerca de 34 mil viajes en 1996, aspecto que está relacionado con la mayor duración de los viajes en la pesquería de la zona centro-sur de Chile (Böhm et al., 1996, 1997; Hernández y Sepúlveda, 1998). A su vez, la capacidad de bodega acumulada y promedio de la flota nacional exhibe un continuo incremento, particularmente desde 1983 a 1996 (Figura 2b).

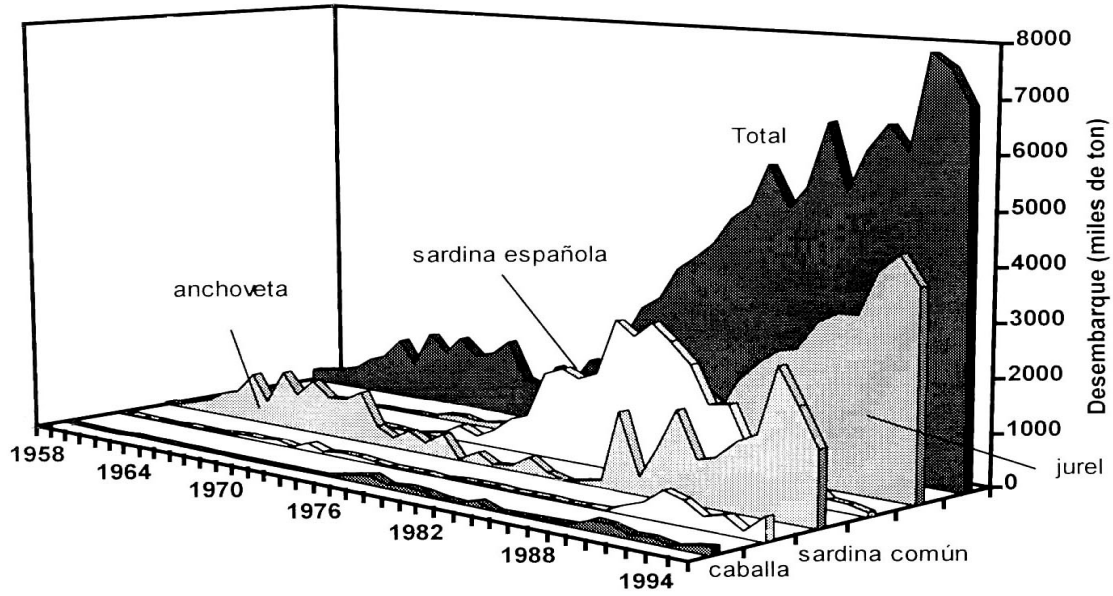

Figura 1. Evolución del desembarque anual de las principales especies de peces pelágicos y desembarque total de recursos marinos de Chile entre 1958 y 1996.

Figure 1. Evolution of annual landings of the main pelagic fish and the total marine catch of Chile between 1958 and 1996. 
La relación entre las capturas totales de peces pelágicos y la capacidad de acarreo de la flota indica que las capturas han respondido al incremento del esfuerzo de pesca (Figura 3). Se destaca que en los primeros años de la década de los años 90 varias pesquerías pelágicas se declaran en estado de explotación plena y no se conceden inscripciones de nuevos permisos de pesca. No obstante el esfuerzo de pesca se desarrolla a través del reemplazo de embarcaciones por embarcaciones de mayor tamaño y autonomía, sumándose a ello el mejoramiento tecnológico en equipos de detección y búsqueda, especialmente en la zona centro-sur. El desarrollo tecnológico incrementa el poder de pesca y la eficiencia de pesca, razón por la cual, nosotros pensamos que los años 1994 a 1996 el esfuerzo de pesca debería estar más desplazado hacia la derecha en el gráfico y es la razón del porque aparecen marcados con un signo de interrogación (Figura 3). Además, las altas capturas de 1994 y 1995 han sido sustentadas básicamente por T. s. murphyi, recurso que exhibió un incremento de $28 \%$ y $39,5 \%$ en 1994 y 1995 , respectivamente en relación con los desembarques promedio del período 1991-1993 (3,2 millones de ton; Figura 1). Este incremento de las capturas de T. $s$. murphyi se debe al desarrollo del esfuerzo de pesca y a la mayor cobertura espacial que ha logrado la flota industrial de cerco de Chile centro-sur en 1994 y 1995 (Böhm et al., 1996, 1997). Sobre la base de estos planteamientos, es probable que las capturas nacionales de peces pelágicos han alcanzado o están cerca del nivel máximo de captura que la totalidad de los stocks de peces pelágicos podrían producir en forma excedentaria.
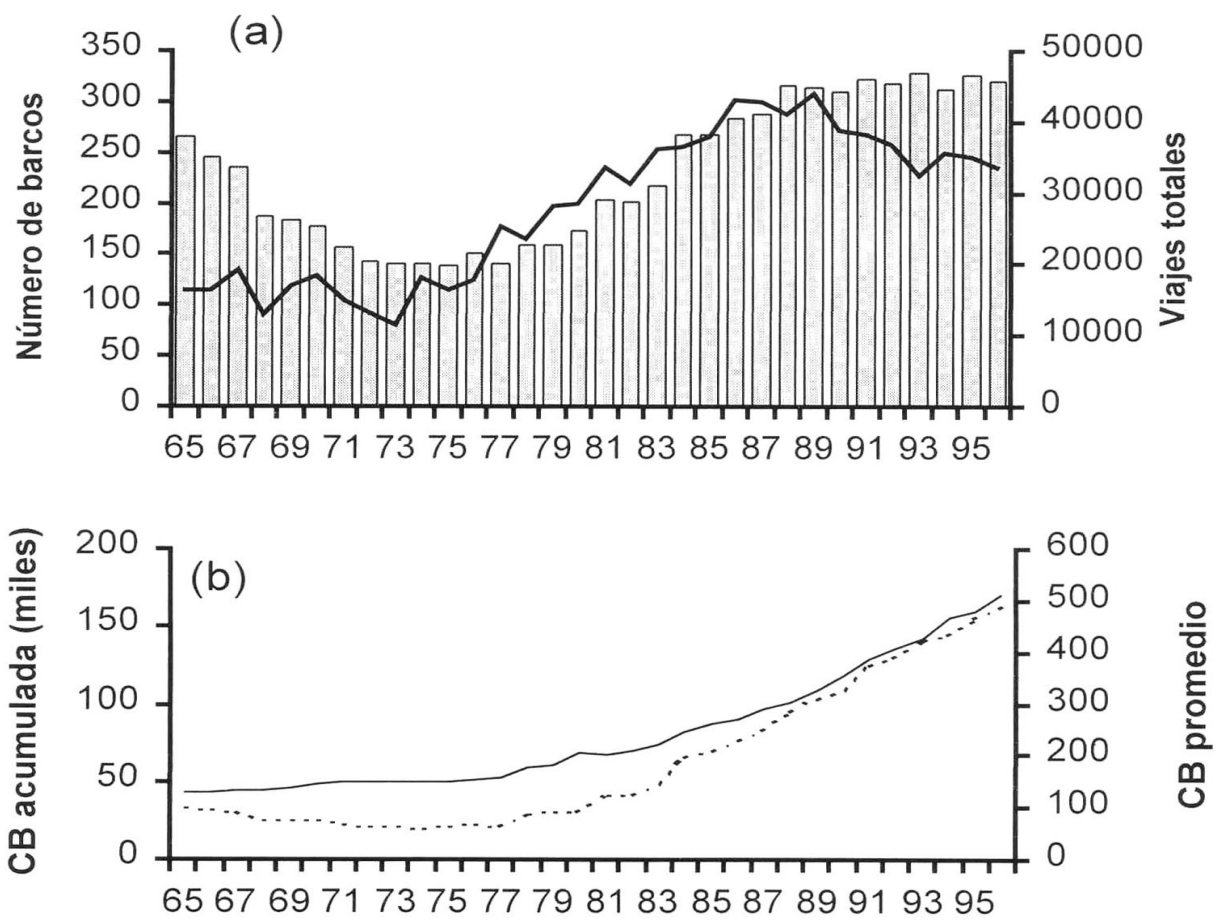

Años

Figura 2. Evolución de la flota nacional entre 1965 y 1996: a) número de embarcaciones (barras) y viajes totales (línea gruesa); b) capacidad de bodega acumulada (línea segmentada) y promedio (línea delgada).

Figure 2. Evolution of the national fleet between 1965 and 1996: a) number of vessels (bars) and total trips (line); b) cumulated hold capacity of fleet (segmented line) and average hold capacity (line). 


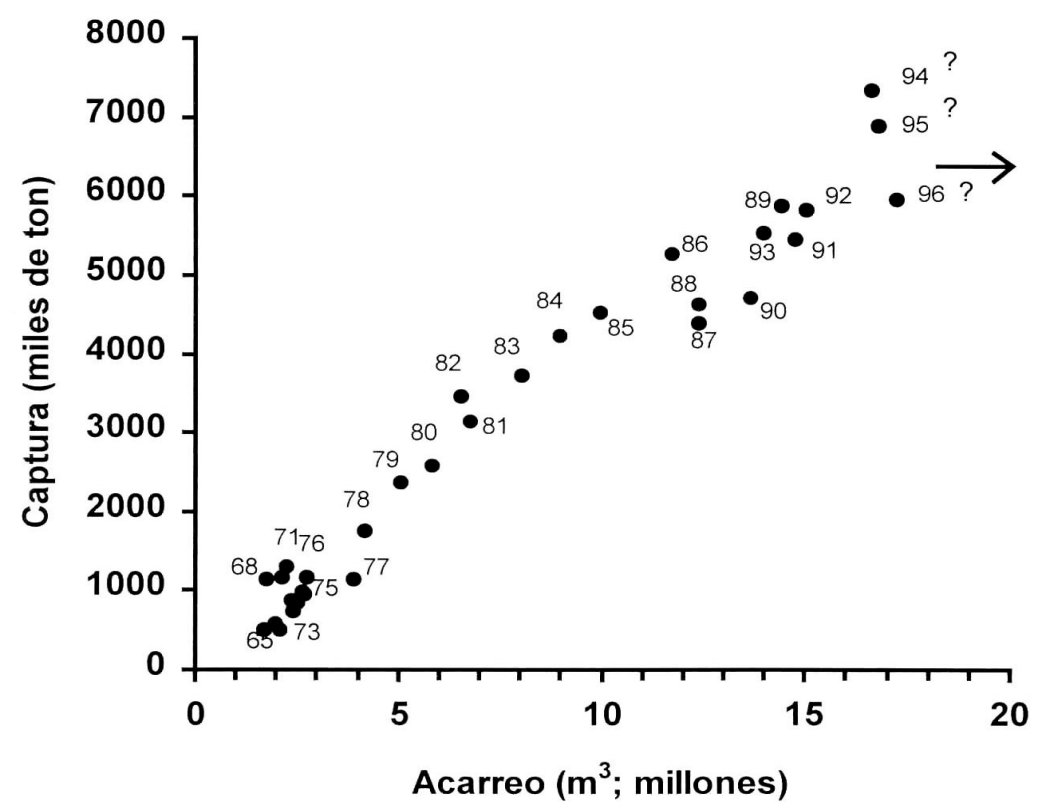

Figura 3. Relación entre las capturas anuales y la capacidad de acarreo de la flota nacional, período $1965-96$. Figure 3. Relationships between the annual catch and the fishing effort of the national fleet, period $1965-96$.

El área costera más productiva, considerada hasta el límite de $60 \mathrm{mn}$ mar afuera, es de $125 \mathrm{mil} \mathrm{Km}^{2}$ para la zona norte (Regiones I a III), 33,6 mil Km² para la zona centro-norte (IV Región), y 87,7 mil $\mathrm{Km}^{2}$ para la zona centro-sur (Regiones V a IX). Si se considera las capturas promedio de peces pelágicos entre 1989 y 1996, la producción primaria requerida (PPR) alcanza valores de 137; 34; y, $614 \mathrm{gCm}^{-2} \mathrm{año}^{-1}$ para las zonas norte, centro-norte y centro-sur, respectivamente (Tabla 1).

La estimación de PPR para la zona centro-sur es muy alta e implica requerimientos de una alta producción primaria (PP). Sin embargo, al considerar la relación de Ware (1992), entre producción primaria y transporte Ekman, se puede tener una idea acerca de la PP anual para cada zona. El transporte Ekman, obtenido de Parrish et al. (1983), corresponden a 0,$31 ; 1,01 ; \mathrm{y}, 0,53$ ton $\mathrm{m}^{-1} \mathrm{~s}^{-1}$ para las zonas norte, centro-norte y centro-sur, respectivamente. Estos valores conducen a estimaciones de PP de $170 \mathrm{gCm}^{-2} \mathrm{año}^{-1}$ para la zona norte, $736 \mathrm{gCm}^{-2} \mathrm{año}^{-1}$ para la zona centro-norte y $348 \mathrm{gCm}^{-2} \mathrm{año}^{-1}$ para la zona centro-sur, las cuales nosotros hemos aproximado a 200, 700 y $350 \mathrm{gCm}^{-2} \mathrm{año}^{-1}$ para cada zona respectivamente. En este contexto, la PP se vería sobrepasada por la PPR solamente en la zona centro-sur de Chile, situación que -como se discute más adelante, podría deberse tanto a una subestimación de la PP para la zona centro-sur como al modo de vida de $T$. s. murphyi; entendiéndose como modo de vida a la trayectoria espacio-temporal determinada, en el tiempo, por el ciclo de vida y en el espacio, por las rutas de migración-dispersión de la especie (Bernal, 1990).

Se debe considerar que en la zona centro-sur el proceso de surgencia costera se presenta estacionalmente, desde inicios de septiembre hasta fines de marzo (Arcos y Navarro, 1986; Arcos, 1987). Esta estacionalidad en la actividad del viento favorable a la surgencia, fue considerada en el promedio del transporte de Ekman de 0,53 ton $\mathrm{m}^{-1} \mathrm{~s}^{-1}$ (Parrish et al., 1983).

La PPR de la zona centro-sur de Chile está influenciada por las altas capturas de T. s. murphyi. Este recurso se caracteriza por ser altamente migratorio y de distribución oceánica, cuyos individuos más longevos han alcanzado incluso las costas de Nueva Zelandia, a lo largo de la corriente de deriva 
Tabla 1. Capturas, nivel trófico, y produción primaria requerida (PPR) por especies y zonas de estudio. Las capturas son el promedio del período 1989-96.

Table 1. Catch, trophic level, and primary production required (PPR) by species and zones. The catches are the mean of the period 1989-96

\begin{tabular}{|c|c|c|c|c|}
\hline AREA & $\begin{array}{l}\text { Captura } \\
\text { niles de ton) }\end{array}$ & $\begin{array}{c}\text { Captura } \\
\text { gCm }^{-2} \mathbf{a n ̃ o} 0^{-1}\end{array}$ & NT & $\begin{array}{c}\text { PPR } \\
\text { gCm }^{-2} a_{\tilde{n} 0^{-1}}\end{array}$ \\
\hline \multicolumn{5}{|c|}{ a) Zona Norte (área= $125000 \mathrm{Km}^{2}$ ) } \\
\hline E. ringens & 1249,2 & 1,11 & 2,6 & 44,2 \\
\hline S. sagax & 566,4 & 0,50 & 2,6 & 20,0 \\
\hline S. japonicus & 104,6 & 0,09 & 3,3 & 18,6 \\
\hline T. s. murphyi & 386,0 & 0,34 & 3,2 & 54,4 \\
\hline Sub-total & 2306,3 & 2,05 & & 137,2 \\
\hline \multicolumn{5}{|c|}{ b) Zona Centro-Norte (área=33600 $\mathrm{Km}^{2}$ ) } \\
\hline E. ringens & 22,5 & 0,08 & 2,6 & 3,0 \\
\hline S. sagax & 20,2 & 0,07 & 2,6 & 2,7 \\
\hline S. japonicus & 1,3 & 0,004 & 3,3 & 0,9 \\
\hline T. s. murphyi & 53,1 & 0,18 & 3,2 & 27,8 \\
\hline Sub-total & 97,1 & 0,32 & & 34,3 \\
\hline \multicolumn{5}{|c|}{ c) Zona Centro-sur (área=87700 Km²) } \\
\hline E. ringens & 282,6 & 0,36 & 2,6 & 14,3 \\
\hline S. sagax & 32,3 & 0,04 & 2,6 & 1,6 \\
\hline S. bentincki & 323,5 & 0,41 & 2,6 & 16,3 \\
\hline S. japonicus & 3,6 & 0,005 & 3,3 & 0,9 \\
\hline T. s. murphyi (a) & 2893,0 & 3,67 & 3,2 & 580,9 \\
\hline T. s. murphyi (b) & 1686,6 & 2,14 & & 338,7 \\
\hline Sub-total(a) & 3534,9 & 4,12 & & 614,0 \\
\hline Sub-total(b) & 2328,5 & 2,95 & & 371,8 \\
\hline TOTAL(a) & 5938,3 & 2,55 & & 292,9 \\
\hline TOTAL(b) & 4732,0 & 2,14 & & 206,7 \\
\hline
\end{tabular}

a considerando la captura promedio de $T$. $s$. murphyi de la zona centro-sur en el período 1989-1996.

${ }^{\mathrm{b}}$ considerando que T. s. murphyi tiene acceso por 7 meses a la productividad de la zona centro-sur (ver texto).

del Oeste (Serra, 1991; Grechina, 1992; Arancibia et al., 1995a). La disponibilidad de T. s. murphyi aumenta notablemente durante la época de otoñoinvierno (marzo-agosto) en la zona centro-sur por razones de engorde. En esta época, la flota industrial de cerco obtiene la más altas capturas y rendimientos (Figura 4); en tanto que, hacia fines de agosto la disponibilidad de $T$. s. murphyi diminuye pro-
Tabla 2. Area productiva costera, producción primaria, producción primaria requerida y eficiencia de rendimiento pesquero de las zonas norte, centro-norte, centro-sur y área total del sistema de surgencia costera de Chile. (a) con la influencia parcial de $T$. $s$. murphyi al sistema costero y (b) sin la influencia de este recurso.

Table 2. Coastal productive area, primary production, primary production required and yield efficiency of northern zone, central-northern zone, centralsouthern zone and total of the Chilean upwelling system. (a) taking into account $T$. s. murphyi as a part of the coastal upwelling system and (b) without the influence of $T$. s. murphyi.

\begin{tabular}{|cl|}
\hline Norte & $\begin{array}{l}\text { Centro Centro Total } \\
\text {-norte -sur }\end{array}$ \\
\hline
\end{tabular}

a) Con T. s. murphyi:

$\begin{array}{lrrrr}\text { Area }\left(\mathrm{Km}^{2}\right) & 125000 & 33600 & 87700 & 246300 \\ \text { Captura }\left(10^{11} \mathrm{gCaño}^{-1}\right) & 2,56 & 0,11 & 3,93 & 6,60 \\ \text { PPR }\left(10^{11} \mathrm{gCaño}^{-1}\right) & 171,49 & 11,54 & 538,48 & 721,51 \\ \text { PPR }\left(\mathrm{gCm}^{-2} \mathrm{año}^{-1}\right) & 137,19 & 34,33 & 614,01 & 292,94 \\ \text { PP }\left(\mathrm{gCm}^{-2} \mathrm{año}^{-1}\right) & 200 & 700 & 350 & 322 \\ \text { \%PP } & 68,60 & 4,90 & 175,43 & 91,08 \\ \text { Eficiencia (\%) } & 1,03 & 0,05 & 1,28 & 0,83\end{array}$

b) Sin T. s. murphyi:

$\begin{array}{lrrrr}\text { Area }\left(\mathrm{Km}^{2}\right) & 125000 & 33600 & 87700 & 246300 \\ \text { Captura }\left(10^{11} \mathrm{gCaño} \mathrm{O}^{-1}\right) & 2,13 & 0,05 & 0,71 & 2,90 \\ \text { PPR }\left(10^{11} \mathrm{gCaño}^{-1}\right) & 103,51 & 2,18 & 29,03 & 134,72 \\ \text { PPR }\left(\mathrm{gCm}^{-2} \mathrm{año}^{-1}\right) & 82,81 & 6,49 & 33,10 & 54,70 \\ \text { PP }\left(\mathrm{gCm}^{-2} \mathrm{ano}^{-1}\right) & 200 & 700 & 350 & 322 \\ \text { \%PP } & 41,40 & 0,93 & 9,46 & 17,01 \\ \text { Eficiencia (\%) } & 0,85 & 0,02 & 0,23 & 0,37\end{array}$

gresivamente debido a procesos de migración fuera de la zona costera para realizar el desove. En este contexto, se puede considerar que $T$. s. murphyi accede parcialmente a la producción biológica de la zona costera de Chile centro-sur. Nosotros estimamos que dicho acceso ocurre aproximadamente durante 7 meses (otoño-invierno; 0,583 año) en la zona centro-sur en base a la estacionalidad de las capturas (Figura 4). Por ende, la reducción de los niveles de desembarque de jurel a 0,583 año conduce a estimaciones de PPR de $372 \mathrm{gCm}^{2} \mathrm{año}^{-1}$ para la zona centro-sur (Tabla 1, Subtotal b).

Se destaca que las capturas de T. s. murphyi tienen una alta incidencia en el cálculo de PPR en todas las zonas de estudio (Tabla 2), aspecto que se 


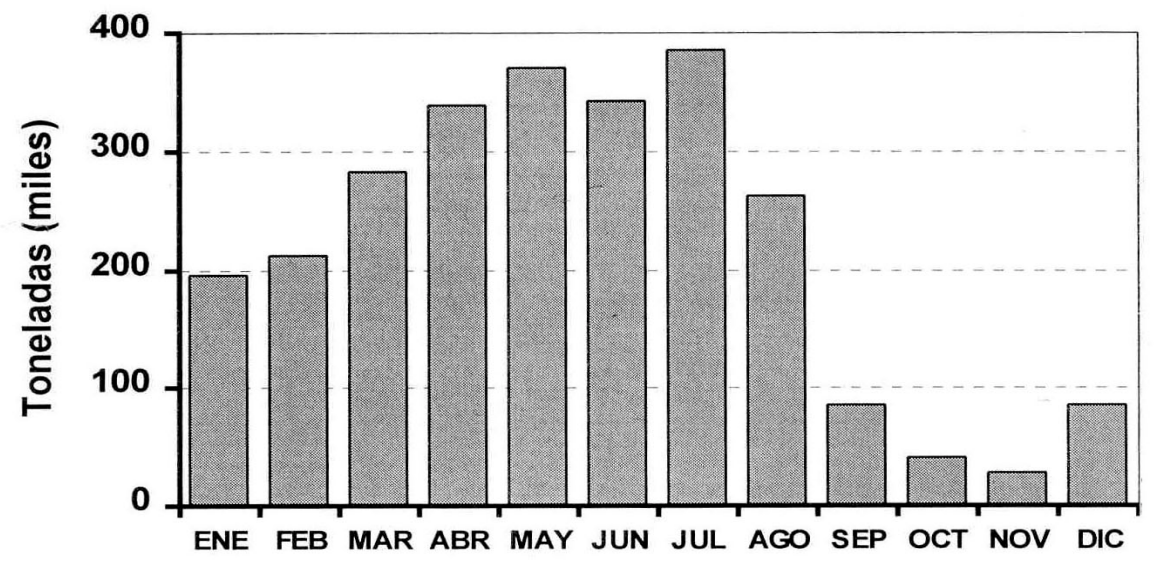

Figura 4. Capturas promedio de T. s. murphyi en la zona centro-sur de Chile, período 1989-96.

Figure 4. Mean catch of T. s. murphyi in the center-south of Chile, period 1989-96.

debe tanto a la más alta posición trófica como a las altas capturas de este recurso en el período 198996.

Las medidas de eficiencia mostradas en la Tabla 2, se definen como la captura dividida por la PP y expresan la fracción de PP que llega a las pesquerías a la forma de captura o excedente de producción de los stocks de peces explotados (Ware, 1992). A nivel nacional, y considerando que T. $s$. murphyi es un componente que tiene un acceso completo al sistema costero, la fracción de la PP que llega a las pesquerías de peces pelágicos alcanza a $0,83 \%$ a nivel nacional; mientras que, dejando fuera al jurel del sistema costero, la eficiencia se reduce a $0,37 \%$. De igual forma, la PPR alcanza a 91\% de la PP cuando $T$. s. murphyi se incluye formando parte del sistema; mientras que baja al $17 \%$ cuando no se considera este recurso (Tabla 2).

\section{DISCUSION}

Los sistemas de surgencia de margen oriental de los océanos se caracterizan por su alta variabilidad (Barber y Smith, 1981) y por ser comparables en términos de la composición de especies de peces (Bakun y Parrish, 1980; Parrish et al., 1983). El análisis de series de tiempo de las capturas de peces pelágicos en estos sistemas muestra que estas pesquerías rara vez se sostienen en niveles productivos por más de 10 a 15 años (Sharp, 1987; Lluch-Belda et al., 1989). En este contexto, surge la necesidad de evaluar la productividad de estos recursos de peces tomando en cuenta la naturaleza variable de las regiones de surgencia y de analizar la probabilidad latente de cambios en la dominancia de especies (Hutchings, 1992).

En general, la producción primaria en sistemas de surgencia se caracteriza por estar dominada por células grandes (e.g. diatomeas), lo que resulta ser la condición más favorable para la exportación y secuestro de carbono biogénico (Legendre y Le Fèvre, 1992). No obstante, desde la perspectiva de las pesquerías, la más importante medida de efectividad de un sistema de surgencia es la cantidad de biomasa de peces que puede ser removida anualmente por la pesca considerando el excedente productivo de los stocks explotados. En este estudio, la metodología que se utilizó para estudiar la producción primaria requerida (PPR) se basa en una descripción cuantitativa de los flujos tróficos, la cual ha sido propuesta por Christensen y Pauly (1993), y utilizada por Pauly y Christensen (1995) para estimar la PPR de la pesquería mundial.

En este trabajo, uno de los más altos valores de PPR (581 $\left.\mathrm{gCm}^{-2} \mathrm{año}^{-1}\right)$ fue estimado para $T . s$. murphyi en la zona centro-sur, lo que da cuenta de cerca de 3,3 millones de ton anuales. Este valor se considera alto y conlleva a plantear la necesidad de conocer estimaciones más precisas y confiables de la productividad primaria de la zona costera a lo largo de Chile. Recientemente se puede observar que se están haciendo esfuerzos necesarios en esa dirección (Montecino et al., 1996; Daneri et al., 1998), y los valores de PP aquí estimados con un alto gra- 
do de incertidumbre, se pueden comparar con los registros de PP comunicadas por estos autores. Daneri et al. (1998), comunican que el rango de la PP integrada medidos en el ecosistema de surgencia de Antofagasta fue de 0,12 a $9,3 \mathrm{gCm}^{-2} \mathrm{~d}^{-1}$, mientras que en invierno se obtuvo un valor integrado de PP de 2,17 $\mathrm{gCm}^{-2} \mathrm{~d}^{-1}$. Nuestra estimación de $200 \mathrm{gCm}^{-2} \mathrm{año}^{-1}$ para la zona norte se encuentra cercana al rango inferior (» $\left.0,55 \mathrm{gCm}^{-2} \mathrm{~d}^{-1}\right)$. Para la zona centro-norte, Daneri et al. (1998) comunican valores integrados de PP entre 1,46 y $2,8 \mathrm{gCm}^{-2} \mathrm{~d}^{-1}$ para la estación COSMOS de Coquimbo, mientras que en aguas más oceánicas (estación OCEMOS) se obtuvo una PP de 0,66 gCm${ }^{2} \mathrm{~d}^{-1}$; similares valores de PP comunican Montecino et al. (1996), i.e. entre 0,14 y $3 \mathrm{gCm}^{-2} \mathrm{~d}^{-1}$ en las estaciones costeras y entre 0,25 y $1,32 \mathrm{gCm}^{-2} \mathrm{~d}^{-1}$ para las estaciones oceánicas. En este estudio se estima una PP de » $1,92 \mathrm{gCm}^{-2} \mathrm{~d}^{-1}$ para la zona centro-norte, valor que se encuentra dentro de los valores de PP registrados. Para la zona centro-sur, Daneri et al. (1998) indican que los valores de PP variaron, en promedio, de 0,5 a $6 \mathrm{gCm}^{-2} \mathrm{~d}^{-1}$ siendo junio, julio y agosto los meses menos productivos $\left(<1 \mathrm{gCm}^{-2} \mathrm{~d}^{-1}\right)$. Sin embargo, en esta zona los autores mencionan que en una oportunidad se registró un valor de PP de $19,9 \mathrm{gCm}^{-}$ ${ }^{2} \mathrm{~d}^{-1}$, uno de los valores más altos registrados. Asimismo, la PP anual para la zona centro-sur ha sido calculada en $800 \mathrm{gCm}^{-2}$ por estos mismo autores, mientras que en este trabajo se utiliza una PP de $350 \mathrm{gCm}^{-2}$.

De acuerdo a lo expuesto, nuestras estimaciones de PP para la zona norte y centro-norte se pueden considerar como valores adecuados, pero conservadores. Sin embargo, en el caso de la zona centro-sur, y de acuerdo con los resultados de Daneri et al. (1998), es probable que nosotros estemos subestimando en cerca de 0,5 veces la magnitud de la PP anual. Aspecto que podría explicar la PPR estimada para la zona en $614 \mathrm{gCm}^{-2} \mathrm{año}^{-1}$. Se destaca que nuestras estimaciones de PP involucran extrapolaciones y un alto grado de incertidumbre, ya que dependen de una relación establecida por Ware (1992), cuyo propósito sólo fue comparativo. Aunque los valores de PP utilizados en este estudio se encuentran dentro de los valores registrados en las diferentes zonas, el propósito de tales estimados son sólo referencias para comparar nuestras estimaciones de PPR.

En este contexto, para sostener la captura nacional de peces pelágicos en un nivel promedio similar al observado en el período 1989-1996 (5,9 millo- nes de ton, Tabla 1), la producción primaria requerida alcanza a $293 \mathrm{gCm}^{-2} \mathrm{año}^{-1}$. Este valor se considera alto si se acepta la estimación de PP para el sistema total (i.e. $322 \mathrm{gCm}^{-2} \mathrm{año}^{-1}$, Tabla 2), y la conclusión más obvia sería que las principales pesquerías nacionales de peces pelágicos habrían llegado al límite de la capacidad de sustentación de la zona costera más productiva. Sin embargo, considerando que es muy probable que la PP estimada para la zona centro-sur esté subestimada en este estudio, al corregir aumentando al doble la PP para esta zona, la PP nacional alcanzaría a $446 \mathrm{gCm}^{-2} \mathrm{año}^{-1} \mathrm{y}$, por lo tanto, la PPR sería de 65,6\% de la PP. En otras palabras, aunque el valor de PP de la zona centro-sur sea corregido, la PPR para sustentar el desembarque nacional de peces pelágicos, en el nivel promedio de 5,9 millones de ton, de todas maneras sigue siendo alta para la capacidad productiva del ecosistema de surgencia costera.

En relación con las medidas de eficiencia de rendimiento pesquero, que expresa la fracción de PP que llega a las pesquerías a la forma de captura o excedente de producción de los stocks de peces explotados, a nivel nacional alcanza a $0,83 \%$; mientras que, dejando fuera al jurel del sistema costero, la eficiencia se reduce a $0,37 \%$ (Tabla 2). Estos valores son muy superiores a los estimados por Ware (1992) para los sistemas de surgencia de Perú, California y Benguela, los cuales fluctúan entre 0,05\% para California, y 0,16\% para Perú. Una explicación de tan altos valores de eficiencia de rendimiento pesquero se puede deber al valor más conservativo de PP que se ha utilizado en este estudio, ya que Ware (1992) utiliza $1.225 \mathrm{gCm}^{-2} \mathrm{año}^{-1}$ para Perú, $735 \mathrm{gCm}^{-2} \mathrm{año}^{-1}$ para Benguela y $343 \mathrm{gCm}^{-2} \mathrm{año}^{-1}$ para California. Si bien es cierto aquí se estima una PP similar a la de California, las capturas de peces pelágicos que se extraen en Chile son notablemente mayores que la de dicho sistema, lo que incide en el cálculo de la eficiencia del rendimiento pesquero.

En la zona norte, la PPR por E. ringens alcanza a $44 \mathrm{gCm}^{-2}$ año ${ }^{-1}$ para un nivel de captura promedio de 1,249 millones de ton. Esta cantidad es razonable si se acepta el nivel de PP de $200 \mathrm{gCm}^{-2} \mathrm{año}^{-1}$ (Tabla 1 y 2). Las capturas de E. ringens han superado los 2 millones de ton en 1994 y 1995, lo que implica duplicar los requerimientos de PP. No obstante, se debe considerar que las capturas de S. sagax han disminuido, lo que de alguna manera podría compensar cualquier incremento relativo de la PPR por $E$. ringens en la zona norte. Se destaca que esta 
región se caracteriza por la ocurrencia casi permanente de la surgencia inducida por el viento durante todo el año (Fuenzalida, 1990; Barbieri et al., 1996). E. ringens se caracteriza por utilizar los centros de surgencia locales y sus áreas adyacentes como hábitat preferido, ocupándolos durante todo su ciclo de vida (Bernal, 1990). En otras palabras, los centros de surgencia son utilizados por E. ringens tanto durante la época de desove, y como áreas de crianza de juveniles y de alimentación de adultos.

Lo anterior contrasta notablemente con el "modo" de vida de T. s. murphyi, que se caracteriza por ser una especie oceánica y migratoria, y cuya PPR alcanza a 54,4 $\mathrm{gCm}^{-2} \mathrm{año}^{-1}$ en la zona norte, superando los requerimientos de E. ringens. Se debe considerar que T. s. murphyi tiene una notable incidencia en los valores de PPR en todas las regiones estudiadas (Tabla 1 y 2). En efecto, si se elimina esta especie del análisis, sólo $55 \mathrm{gCm}^{-2} \mathrm{anono}^{-1}$ de la PP se requiere para la sustentabilidad de las pesquerías del resto de los peces pelágicos a nivel nacional (Tabla $2 b$ ), solamente $17 \%$ si se acepta el valor de PP estimado en $322 \mathrm{gCm}^{-2} \mathrm{año}^{-1}$ para el país. Esta fracción de la PP (17\%), aparece siendo más bajo que el obtenido por Pauly y Christensen (1995) para todos los sistemas de surgencia del mundo (i.e. $25 \%$ de la PP), pero conlleva necesariamente dejar fuera del sistema al recurso jurel, principal recurso pelágico que actualmente se extrae en Chile.

En la zona centro-sur, la PPR por T. s. murphyi, según niveles de captura de 1,7 millones de ton $\left(\mathrm{PPR}=339 \mathrm{gCm}^{-2} \mathrm{año}^{-1}\right.$ ) ó 2,9 millones de ton $\left(\mathrm{PPR}=581 \mathrm{gCm}^{-2} \mathrm{año}^{-1}\right)$, la primera considerando que el recurso accede a la PP de la región costera sólo 7 meses en el año en la zona centro-sur (febrero-agosto) y la segunda como el promedio de captura total en el período 1989-96, se puede justificar dada la posición trófica más alta de esta especie, requiriendo por esta razón una mayor fracción de la producción primaria que los clupeiformes, por ejemplo. En efecto, los recursos pelágicos netamente neríticos y costeros, como E. ringens y S. bentincki que corresponden a las especies que ocupan el segundo lugar en capturas en la zona centro-sur, sólo requieren en total cerca de $30 \mathrm{gCm}^{-2} \mathrm{año}^{-1}$, aproximadamente $8 \%$ de la PP estimada para la región $\left(350 \mathrm{gCm}^{-2} \mathrm{año}^{-1}\right.$, Tabla 1 y 2).

Considerando que en la zona centro-sur la surgencia inducida por el viento presenta un comportamiento marcadamente estacional, lo que incide en la ocurrencia de un máximo de producción estival, debería existir algún componente biológico que permita distribuir y canalizar la productividad de la región hacia niveles tróficos intermedios como T. s. murphyi, Merluccius gayi y otras especies ícticas durante el resto del año, fuera de la estación de máxima producción primaria.

Sin duda los eufáusidos, y particularmente Euphausia mucronata, deben cumplir un rol significativo en la estructuración y la dinámica del ecosistema de surgencia de Chile centro-sur. En efecto, los eufáusidos poseen varias características las cuales contribuyen a su importancia en estos sistemas, las cuales se pueden resumir en: (a) alimentación omnívora, lo que les permite acceder y utilizar una gran variedad de oferta ambiental de alimento, y por ende acoplarse a las fluctuaciones ambientales de alimento; (b) alcanzan tamaños más grandes y las expectativas de vida son mayores (»1 año) que otros componentes del zooplancton; (c) tienden a formar grandes agregaciones, lo que permite que sean accesibles a una amplia variedad de predadores a través del año; (d) la producción de huevos es invernal, justo antes de la ocurrencia de eventos de surgencia en primavera y, por ende, existe un acoplamiento al incremento en la biomasa fitoplanctónica. Estas características permiten que los eufáusidos puedan ser capaces de suavizar el efecto de la variabilidad estacional, permitiendo canalizar y distribuir la energía hacia niveles tróficos superiores durante todo el año (Pillar et al., 1992). Además existen evidencias que sugieren que los eufáusidos juegan un rol en la regulación de la producción y biomasa del mesozooplancton, más bien que sobre los productores primarios (Stuart y Pillar, 1990).

Diversos análisis de la alimentación de $T$. $s$. murphyi, revelan que los eufáusidos constituyen el item alimentario más importante con más del $90 \%$ en peso, particularmente en la época de otoño-invierno, mientras que hacia primavera aumenta la proporción de peces mesopelágicos y otros componentes del macrozooplancton (Cornejo, 1991; Arancibia et al., 1995b, Miranda et al., 1998). En este contexto, y considerando que $T$. s. murphyi es una especie oceánica migratoria, ésta sólo accede a la productividad de la zona costera predando sobre los eufáusidos por razones de engorde (Quiñones et al., 1997). Siguiendo a Runge (1988), lo anterior conlleva a plantear la hipótesis de "débil conexión/ fuerte filtro", en otras palabras existe una débil conexión entre la producción primaria de la zona costera en Chile centro-sur con la disponibilidad de $T$. 
s. murphyi, pero el impacto de las fluctuaciones en la productividad primaria es "filtrada" por la dinámica de las poblaciones micronectónicas. Quiñones et al. (1997) indican que T. s. murphyi puede encontrar agregaciones de alimento (i.e. eufáusidos) sin mayor dificultad en la zona centro-sur durante la época invernal, lo que podría ser favorable para la engorda de los peces. Las razones para pensar en un período de engorda se basan en el supuesto de que los gastos metabólicos asociados con la migración y la reproducción (desove) en primavera deban ser altos. Por otra parte, el período de engorda en la zona costera debería ser más importante para los peces jóvenes, más productivos desde el punto de vista de su aporte per cápita a la reproducción, los cuales deben acumular reservas antes de iniciar la emigración primaveral hacia la zona oceánica para desovar. En consecuencia, el acceso de T. s. murphyi a la productividad de la región costera debe necesariamente ser parcial ya que por su condición de especie oceánica y migratoria es probable que pueda acceder a otros sistemas productivos en primaveraverano, tal como divergencias oceánicas, frentes oceánicos, y otras estructuras de mesoescala de naturaleza transiente asociadas a la convergencia subantártica (Vinogradov et al., 1990).

Sobre la base de los antecedentes expuestos, y en relación con la sustentabilidad de las pesquerías de peces pelágicos por la capacidad productiva del sistema de surgencia costera de Chile, al parecer se ha llegado a un nivel muy cercano al máximo sustentable ya que las capturas han tendido a estabilizarse a partir de 1990, mientras que el poder de pesca de la flota sigue incrementándose a través de mejoras tecnológicas y reemplazo de embarcaciones pequeñas por otras de mayor tamaño. Aunque se debe reconocer que esta afirmación no es muy es evidente desde la relación existente entre las capturas y el esfuerzo de pesca de la flota nacional, probablemente debido a la influencia de las altas capturas de E. ringens y T. s. murphyi en 1994 y 1995 y al hecho que se ha utilizado una medida de esfuerzo que no recoge la influencia del mayor poder de pesca de la flota asociado al desarrollo tecnológico. En efecto, tal vez si se pudiera estandarizar el esfuerzo de pesca de la flota nacional, corrigiendo los poderes de pesca por el desarrollo tecnológico, entonces el «plateau» alcanzado por las capturas pueda ser más evidente. Se destaca que en pesquerías multiespecíficas usualmente se utiliza el modelo de producción de Schaefer (1954), ya que requiere una cantidad mínima de información para evaluar la productividad de los stocks explotados (i.e. capturas y esfuerzo de pesca totales). Cuando en un modelo de producción se utiliza las capturas totales de más de una especie, generalmente se observa una mejor descripción de la situación de la explotación que cuando se aplica el mismo modelo a uno de los varios stocks que componen la captura total. Al parecer, las razones de un mejor ajuste se deben a que la biomasa total reacciona de una manera simple al esfuerzo de pesca total aplicado o bien porque en el análisis se promedia varios procesos, siendo esta última la explicación más razonable (FAO, 1978; Pauly, 1979).

En consecuencia, sobre la base de los criterios analizados en este estudio, se plantea que la capacidad productiva del ecosistema marino costero de Chile limita el desembarque nacional de peces pelágicos costeros. Además, la sustentación de niveles de desembarque como los del promedio del período 1989-96, sólo es posible si los excedentes productivos del stock de $T$. $s$. murphyi-que por su naturaleza migratoria puede acceder a otros sistemas productivos- permiten mantener la intensidad de explotación actual ejercida por la flota industrial de cerco de la zona centro-sur.

\section{AGRADECIMIENTOS}

Los autores agradecen la revisión y crítica de este trabajo por parte de dos revisores anónimos.

\section{REFERENCIAS}

Ahumada, R. y L. Chuecas. 1979. Algunas características hidrográficas de la bahía de Concepción y áreas adyacentes, Chile. Gayana Misc., 8: 1-56.

Ahumada, R. 1989. Producción y destino de la biomasa fitoplanctónica en un sistema de bahías en Chile central: una hipótesis. Biología Pesquera 18: 53-66.

Arancibia, H.; L. Cubillos, D. Arcos, A.S. Grechina y L. Vilugrón. 1995a. The fishery of horse mackerel (Trachurus symmetricus murphyi) in the south Pacific Ocean, with notes on the fishery off central-southern Chile. In: International symposium on middle-sized pelagic fish, C. Bas, J.J. Castro y J.M ${ }^{\text {a }}$. Lorenzo (Eds.), Scientia Marina 59(3-4): 589-596. 
Arancibia, H.; L. Miranda y R. Alarcón. 1995b. Evaluación hidroacústica de jurel en la zona centro-sur, V a IX Regiones, Sección trofodinámica. R. Quiñones, H. Muñoz, R. Serra, S. Núñez, J. Córdova y D. Figueroa. Informe Final Proyecto FIP, 221 p. +285 figs.

Arcos, D. y N. Navarro. 1986. Análisis de un índice de surgencia para la zona de Talcahuano, Chile (Lat. $37^{\circ}$ S).Investigación Pesquera (Chile), 33:9198 .

Arcos, D. 1987. Seasonal and short time scale variability in copepod abundance ans species composition in an upwelling area off Concepción coast, Chile. Ph.D. Thesis Dissertation, State University of New York, Stony Brook, 203 p.

Bakun, A. y R.H. Parrish. 1980. Environmental input to fishery population models for eastern boundary current regions. In: G.D. Sharp (Ed.), Workshop on the effects of environmental variation on the survival larvel pelagic fishes. IOC Workshop Rep. $\mathrm{N}^{\circ} 28$, p. 67-104.

Barber, R.T. y R.L. Smith. 1981. Coastal upwelling ecosystem. In: Analysis of marine ecosystem. A.R. Longhurst (Ed.). London, Academic Press, p. 3168.

Barbieri, M.A., M. Bravo, M. Farías, A. Gonzalez, O. Pizarro y E. Yáñez. 1995. Fenómenos asociados a la estructura térmica superficial del mar observados a través de imágenes satelitales en la zona norte de Chile. Invest. Mar., Valparaíso, 23: 99122.

Bernal, P. 1990. La oceanografía del sistema de corrientes de Chile-Perú en relación a las pesquerías pelágicas: una revisión. In: Perspectivas de la actividad pesquera en Chile. M.A. Barbieri (Ed.). Escuela de Ciencias del Mar, UCV, p. 35-48.

Böhm, M.G., M.A. Barbieri, E. Yáñez, V. Catasti y A. González. 1996. Análisis de la captura y del esfuerzo de pesca de las unidades de pesquería de jurel de la zona centro-sur y norte. Informe Final Proyecto FIP N ${ }^{\circ}$ 25-94. Est. y Doc., Escuela de Ciencias del Mar, UCV, Valparaíso, 133 pp.

Böhm, M.G., M.A. Barbieri, G. Rosson, E. Yáñez, V. Catasti, A. González, L. Cubillos, A. Sepúlveda, A. Hernández y P. Sobarzo. 1997. Análisis de la captura y del esfuerzo de pesca en la pesquería de jurel de la zona centro-sur (V a IX Regiones). Informe Final Proyecto FIP N ${ }^{\circ}$ 96-18. Instituto de Fomento Pesquero, Fondo de Investi- gación Pesquera, Valparaíso, 81 p.

Caballero, G.L.; L. Santillán y G. Rosson. 1992. Investigación del esfuerzo pesquero en las principales pesquerías chilenas: pesquerías pelágicas. CORFO-IFOP (SGI-IFOP 92/16), Instituto de Fomento Pesquero, Santiago, 38 p.

Cáceres, M. y D. Arcos. 1991. Variabilidad en la estructura espacio-temporal de un área de surgencia frente a la costa de Concepción, Chile. Invest. Pesq. (Chile) 36:27-38.

Christensen, V. y D. Pauly, 1993. Flow characteristics of aquatic ecosystems. In: Trophic models of aquatic ecosystems, V. Christensen y D. Pauly (Eds.), ICLARM Conf. Proc. 26, p. 338-352.

Christensen, V. y D. Pauly. 1995. Fish production, catches and the carrying capacity of the world oceans. NAGA, the ICLARM Quarterly 18(3): 3440.

Cornejo, M.H. 1991. Causas posibles de las agregaciones de jurel (Trachurus symmetricus murphyi, Nichols): disponibilidad de alimento. Tesis Magister en Ciencias (mención oceanografía), Universidad de Concepción, 108 p.

Cushing, D.H. 1971. Upwelling and the production of fish. Adv. Mar. Biol. 9: 255-334.

Daneri, G., V. Dellarossa, R. Quiñones, B. Jacoby, P. Montero. 1998. Mediciones de productividad primaria y respiración comunitaria en el sistema chileno de la corriente de Humboldt y áreas oceánicas asociadas. In: anexos Resúmenes XVIII Congreso de Ciencias del Mar, Soc. Chilena de Ciencias del Mar, Universidad Arturo Prat, Iquique, 223 p. + anexos.

FAO, 1978. Some scientific problems of multi-species fisheries. Report of the Expert Consultation on Management of Multi-species Fisheries. FAO Fish. Tech. Pap. 181, 42 p.

Fuenzalida, R. 1990. Variabilidad temporal de un índice de surgencia para la zona de Iquique (Lat. $20^{\circ}$ S). Invest. Cient. y Tec., Serie: Ciencias del Mar, 1: 37-47.

Grechina, A.S. 1992. Historia de investigaciones y aspectos básicos de la ecología del jurel, Trachurus symmetricus murphyi (Nichols), en el Pacífico Suroriental. Doc. Téc. Inst. Invest. Pesq. (IIP), 
Talcahuano, 1(2):1-47 + 21 figs.

Hernández, A. y A. Sepúlveda. 1998. Análisis del esfuerzo y rendimiento de pesca de la pesquería del jurel en la zona centro-sur de Chile. In: «Biología y ecología del jurel en aguas chilenas», D. Arcos (Ed.), Instituto de Investigación Pesquera, Editora Aníbal Pinto, Concepción, p. 191-199.

Hutchings, L. 1992. Fish harvesting in a variable, productive environmental -searching for rules or searching for exceptions? In: Benguela trophic functioning. A.I.L. Payne, K.H. Brink, K.H. Mann y R. Hilborn (Eds.), S. Afr. J. mar. Sci. 12: 297318.

Jarre, A., P. Muck y D. Pauly. 1989. Interactions between fish stocks in the Peruvian upwelling ecosystem. ICES MSM Symposium Paper N ${ }^{\circ} 27$, $24 \mathrm{p}$.

Legendre, L. y J. Le Fèvre. 1992. Interactions between hydrodynamics and pelagic ecosystem: relevance to resource exploitation and climate change. In: Benguela trophic functioning. A.I.L. Payne, K.H. Brink, K.H. Mann y R. Hilborn (Eds.), S. Afr. J. mar. Sci. 12: 477-486

Lieth, H. 1978. Patterns of primary production in the biosphere. Dowden, Hutchinson y Ross, Inc., Stroudsburg, Pennylvania, p. 277-282.

Lluch-Belda, R., J.M. Crawford, T. Kawasaki, A.D. MacCall, R.H. Parrish, R.A. Schwartzlose y P.E. Smith. 1989. World-wide fluctuations of sardine and anchovy stocks: the regime problem. S. Afr. mar. Sci. 8: 195-205.

Medina, M. 1992. Interacciones tróficas entre el jurel (Trachurus murphyi Nichols) y la caballa (Scomber japonicus Jordan y Hubbs) en el ecosistema pelágico de la zona norte de Chile. Tesis Biología Pesquera, Departamento de Ciencias del Mar, Universidad Arturo Prat, Iquique, 76 p.

Miranda, L., A. Hernández, A. Sepúlveda, y M. Landaeta. 1998. Alimentación de jurel y análisis de la selectividad en la zona centro-sur de Chile. In: «Biología y ecología del jurel en aguas chilenas», D. Arcos (Ed.), Instituto de Investigación Pesquera, Editora Aníbal Pinto, Concepción, p. 173-187.

Montecino, V., G. Pizarro y D. Quiroz. 1996. Dinámica fitoplanctónica en el sistema de surgencia frente a Coquimbo $\left(30^{\circ} \mathrm{S}\right)$ a través de la relación funcional entre fotosíntesis e irradianza (P-I).
Gayana Oceanol. 4(2): 139-151.

Parrish, R.H.; A. Bakun, D.M. Husby y C.S. Nelson. 1983. Comparative climatology of select environmental processes in relation to eastern boundary currente pelagic fish reproduction. In: Proceedings of the expert consultation to examine changes in abundance and species composition of neritic fish resources. G.D. Sharp y J. Csirke (Eds.), FAO Fish. Rep., 291, p. 731-778.

Pauly, D. 1979. Theory and management of tropical multispecies stocks: a review, with emphasis on the Southeast Asian demersal fisheries. ICLARM Studies and Reviews $\mathrm{N}^{\mathrm{o}} 1,35 \mathrm{p}$.

Pauly, D. y V. Christensen. 1995. Primary production required to sustain global fisheries. Nature 374:255-257.

Pillar, S.C., V. Stuart, M. Barange y M.J. Gibbons. 1992. Community structure and trophic ecology of euphausiids in the Benguela ecosystem. In: Benguela trophic functioning. A.I.L. Payne, K.H. Brink, K.H. Mann y R. Hilborn (Eds.), S. Afr. J. mar. Sci. 12:393-409.

Pizarro, O., S. Hormazábal, A. González y E. Yáñez. 1994. Variabilidad del viento, nivel del mar y temperatura en la costa norte de Chile. Invest. Mar., Valparaíso, 22: 83-101.

Quiñones, R., R. Serra, S. Núñez, H. Arancibia, J. Córdova y F. Bustos. 1997. Relación espacial entre el jurel (Trachurus symmetricus murphyi) y sus presas en la zona centro-sur de Chile. In: Gestión de Sistemas Oceanográficos del Pacífico Oriental, E. Tarifeño (Ed.), Comisión Oceanográfica Intergubernamental de la Unesco. IOC/INF-1046, p. $187-202$.

Runge, J.A. 1988. Should we expect a relationship between primary production and fisheries? The role of copepod dynamics as a filter of trophic variability. In: Biology of copepods. G.A. Boxshall y H.K. Schminke (Eds.). Hydrobiologia 167/168: 61-71.

Ryther, J.H. 1969. Photosynthesis and fish production in the sea. Science 166: 72-76.

Schaefer, M.B. 1954. Some aspects of the dynamics of populations important to the management of the commercial marine fisheries. Inter. Amer. Trop. Tuna Comm. Bull. 1(2): 27-56.

Serra, R. 1986. Desarrollo de la pesquería de anchoveta (Engraulis ringens) y los cambios de 
su abundancia. Invest. Pesq. (Chile) 33: 13-24.

Serra, R. 1991. Important life history aspects of the Chilean jack mackerel, Trachurus symmetricus murphyi. Invest. Pesq. (Chile) 36:67-83.

Serra, R. y I. Tsukayama. 1988. Sinopsis de datos biológicos y pesqueros de la sardina, Sardinops sagax, en el Pacífico Suroriental. FAO, Sinop. Pesca (13) Rev. 1, 60 p.

Sharp, G.D. 1987. Climate and fisheries: cause and effect or managing the long and short of it all. In: The Benguela and comparable ecosystem. A.I.L. Payne, J.A. Gulland y K.H. Brink (Eds.). S. Afr. J. mar. Sci., 5: 811-838.

Stuart, V. y S.C. Pillar. 1990. Diel grazing patterns of all ontogenetics stages of Euphausia lucensand in situ predation rates on copepods in the southern Benguela upwelling region. Mae. Ecol. Prog. Ser.
64: $227-241$.

Vinogradov, M.E.; E.A. Shushkina \& S.A. Evseenko. 1990. Biomasa del plancton forrajero y recursos potenciales del jurel peruano en la zona subantártica del Pacífico. Voprosy Iktyologii 30(6): 1036-1040 (en ruso).

Ware, D.M.1992. Production characteristics of upwelling systems and the trophodynamic role of hake. In: Benguela trophic functioning. A.I.L. Payne, K.H. Brink, K.H. Mann y R. Hilborn (Eds.), S. Afr. J. mar. Sci. 12: 501-513.

Yáñez E., M. García y M.A. Barbieri. 1997. Fluctuaciones de pesquerías pelágicas y del medio ambiente en el Pacífico suroriental. In: E. Tarifeño (ed.) Gestión de Sistemas Oceanográficos del Pacífico Oriental. Comisión Oceanográfica Intergubernamental de la UNESCO. IOC/INF-1046, p. 219-232. 